Article

\title{
Using Piecewise Linearization Method to PCS Input/Output-Efficiency Curve for a Stand-Alone Microgrid Unit Commitment
}

\author{
Ha-Lim Lee ${ }^{1, *(1)}$ and Yeong-Han Chun ${ }^{2}$ \\ 1 Smart Distribution Research Center, Advanced Power Grid Research Division, Korea Electrotechnology \\ Research Institute, bulmosan-ro 10beon-gil, Seongsan-gu, Changwonsi, Gyeongsangnam-do 51543, Korea \\ 2 Power System Lab, Electronic \& Electrical Enginerring School, Hong-Ik University, 94, Wauson-ro, Mapo-gu, \\ Seoul 04066, Korea; yhchun@hongik.ac.kr \\ * Correspondence: halim@keri.re.kr; Tel.: +82-10-9632-2894
}

Received: 7 September 2018; Accepted: 13 September 2018; Published: 17 September 2018

\begin{abstract}
When operating a stand-alone micro grid, the battery energy storage system (BESS) and a diesel generator are key components needed in order to maintain demand-supply balance. Using Unit Commitment (UC) to calculate the optimal operation schedule of a BESS and diesel generator helps minimize the operation cost of the micro grid. While calculating the optimal operation schedule for the microgrid, it is important that it reflects the actual characteristics of the implanted devices, in order to increase the schedule result accuracy. In this paper, a piecewise linearization, on the actual power conditioning system (PCS) input/output-efficiency characteristic curve, has been considered while calculating the optimal operation schedule using UC. The optimal schedule result calculated by the proposed method has been examined by comparing the schedule calculated by a fixed input/output-efficiency case, which is conventionally used while solving UC for a stand-alone microgrid.
\end{abstract}

Keywords: battery energy storage system; micro grid; MILP; PCS efficiency; piecewise linear techniques; renewable energy sources; optimal operation; UC

\section{Introduction}

A microgrid is a power system covering a partial area, which supplies electric power by utilizing a mixture of energy storage systems and distributed generators, such as renewable energy. To increase the usage of renewable energy in a small sized stand-alone microgrid, the capacity of renewable energy should be relatively larger than a normal microgrid. In these power systems, the output fluctuation from the renewable source is large, which causes many electrical problems. To solve and prevent these problems, various issues must be covered [1]. Using a battery energy storage system (BESS) is a possible method to prevent energy waste by charging the over-generated energy produced from the renewable energy sources [2,3]. Therefore, in a stand-alone microgrid, if a BESS is implanted, the availability of renewable sources and the reliability of power system could be increased $[4,5]$. To maintain this characteristic of the microgrid by using diesel generator and a frequency controlling BESS, an optimal operation method is necessary. In this case, in order to synchronize every device installed in the microgrid, such as diesel generator, wind turbine, photovoltaic device, etc., the BESS must be used as the master source, operating under constant voltage and constant frequency mode [6].

Many studies have been done on the stable operation of stand-alone microgrids, in order to efficiently utilize the implanted BESS. The research conducted in $[6,7]$ have proposed a management system using a decision tree, based on real time measured state of charge (SOC) for a grid-connected 
microgrid having a BESS. A management system of a microgrid that operates on grid-connected mode at normal occasions, and stand-alone mode when an accident occurs by controlling the BESS, diesel generators, and hydro-generators is proposed in [8]. In the research conducted in [9,10], an unit commitment (UC) is proposed for a grid-connected microgrid having micro turbines and fuel cells. [11] proposed a mixed integer linear program (MILP) based on rolling horizon controllers for a management system of microgrids having a BESS and renewable sources. In [12], a model predictive controller is proposed for a grid-connected microgrid having a BESS as a management system. The research conducted in [13-15] proposed a MILP calculation method considering a modified optimal dispatch strategy as a management system for a micro grid having a BESS. [16] has proposed a MILP approach for residential distributed energy system planning. [17] proposed an energy management strategy for both grid-connected and isolated microgrids, using a simplified MILP formulation as an UC problem. The research conducted in [18] proposed a MILP approach without using complex heuristics or decompositions for the operation scheduling of a microgrid. [19] proposed a linear programming cost minimization model of UC for stand-alone microgrids in the UK. In [20], an improved genetic algorithm-based method is proposed for UC in a stand-alone microgrid. A mathematical model of frequency control in stand-alone microgrids, which is integrated into the UC problem, is proposed in [21]. The research conducted in [22-24] proposed an optimization methodology for a day-ahead UC model in a microgrid.

This paper regards the actual performance characteristics of a power conditional system (PCS) while drawing the microgrid operation schedule, considering the accuracy of modeling and calculations. Therefore, this paper has assumed that drawing the generator and load device's optimal operation schedule is the most economic and safe method for operating the stand-alone microgrid effectively. In particular, using the MILP method to calculate the UC is assumed to be the most accurate after analyzing research on the related issues. The papers mentioned above have assumed that the efficiency of a PCS, which is connected in front of the BESS, is fixed, whether the input/output quantity from the PCS is small or large. However, the actual PCS efficiency is low when the input/output is small, and the efficiency is high when the input/output is large. In other words, when the input/output from the actual PCS is small, the produced electric loss rate is higher during the power conversion process, compared to larger input/output from the PCS. Ignoring such input/output-efficiency characteristics of the PCS, the precise amount of power loss inccurred from the PCS is disregarded during calculation of $\mathrm{UC}$, and also has possibility for operation failure, when the device implanted in the microgrid operate under the calculated schedule. Also, if the accurate amount of power loss caused from the PCS is not reflected, the total operation cost of the diesel generator could increase compared to the schedule, or cause an imbalance between power supply and demand. In order to compensate for such defects, this paper suggests considering PCS efficiency characteristics, by using piecewise linearization on the actual input/output-efficiency curve of PCS, while calculating UC for the optimal operation schedule. This will reduce the calculation difference of the actual power loss occurred from the PCS, which increases the accuracy of the optimal operation schedule calculated by UC. Such concepts have been developed in an effort to increase the accuracy of a calculated UC schedule, by using the actual device performance characteristics.

The remainder the paper is as follows. In Section 2, the objective functions, constraint conditions, and piecewise linearization of PCS input/output-efficiency for stand-alone microgrid UC calculation are explained. Section 3 contains case study results that has been done in order to analyze the effect of piecewise linearization of PCS input/output-efficiency while calculating the microgrid UC, at an equivalent power system of South Korea's first "energy island", Ga-sa Island.

\section{Formulation of Microgrid UC for Optimal Operation}

In this chapter, the overall outline of this paper will be explained first. The optimization formulation will follow, in order of objective functions and constraints. Applying a piecewise 
linearization method to the input/output-efficiency curve of PCS is explained in a separate section, as it is the key idea of this paper.

\subsection{Overall Outline}

The proposed UC assumes that the target power system is a stand-alone microgrid in an island area, composed of a single BESS and diesel generator, several wind turbines, and photovoltaic devices. The BESS runs on constant voltage constant frequency (CVCF) mode, and the diesel generator is a backup source, which runs only when the electric power is insufficient in the grid. In the proposed stand-alone microgrid UC, the optimal operation schedule of the diesel generator and BESS for the next $24 \mathrm{~h}$ is calculated based on the forecasting data of renewable energy and load. The purpose is to calculate the UC for every $15 \mathrm{~min}$ and renew the $24 \mathrm{~h}$ operation schedule. However, in this paper, only a snapshot view is analyzed to concentrate more on the piecewise linearization of the actual PCS efficiency characteristics. The goal of the UC is to optimize the operation schedule of the distributed energy resources to minimize the diesel generator's operation cost using MILP. In the results of the calculation, the on/off status, output power of the diesel generator, and the SOC of the BESS are calculated. Since power loss occurs from the PCS, it has to be considered while calculating UC by also considering the input/output efficiency of the PCS. If the power loss incurred from the PCS is inaccurate, the devices implanted in the microgrid cannot operate according to the calculated schedule. Therefore, to reduce the difference between the calculated operation schedule and actual performance of the PCS and other devices, the input/output-efficiency characteristics must be considered, by applying a piecewise linearization method to the actual PCS efficiency curve. While solving the UC problem, the MILP technique is used, since several binary variables, such as on/off status of the diesel generator, must be optimized. The objective function and constraints of the proposed stand-alone microgrid UC are as follows.

\subsection{Objective Function}

The objective function of the proposed stand-alone microgrid UC is to minimize the summation of start-up cost and fuel cost of the diesel generator:

$$
\left.\min \left\{\sum_{i=1}^{I} \sum_{t=1}^{T}\left[F L C_{i, t}+S T C_{i, t}\right]-\alpha_{i} \cdot S O C_{i, T}\right\}\right\}
$$

Fuel cost function $F L C_{i, t}$ is defined as the following Equation (2).

$$
F L C_{i, t}=a_{i} g_{i, t}^{2}+b_{i} g_{i, t}+c_{i}
$$

The equation above represents the relation between diesel generator's generation amount and fuel cost in a curve format. However, in order to use it for mathematic calculation, the curve must be approximated into a linear format for each section. $g_{i, t}$, the output of diesel generator $i$, must be divided to match the number of section $b$ as Equation (3).

$$
g_{i, t}=g_{i}^{m} \cdot u_{i, t}+\sum_{b=1}^{B} g_{i, t, b}
$$

The linearized fuel cost for each section is represented as Equation (4).

$$
F L C_{i, t}=F L C_{i}^{m} \cdot u_{i, t}+\sum_{b} M C_{i, b} \cdot g_{i, t, b}
$$


The start-up cost of the diesel generator is calculated only when the status changes from stop (off) to run (on), according to the following equation.

$$
S T C_{i, t}=s_{i, t} \times S T F_{i}
$$

If the total amount of electric power generated from the renewable sources is greater than the total amount of load for the next $24 \mathrm{~h}$, the result of the MILP optimal calculation gives an infeasible solution. If the final value of the SOC exceeds the maximum BESS capacity, which is usually $100 \%$, the operator should rather reduce the charging power or increase the discharging power of the BESS. However, whereas the SOC remains below the maximum capacity, it is better to charge as much as power in the BESS for the next day's operation schedule, since the possibility of running a diesel generator declines. To ensure such operation, $-\alpha_{i} \cdot s o c_{i, T}$ is added in the objective function. It induces the maximization of the SOC at the final time period by charging the over-generated electric energy generated from the renewable source. $\alpha_{i}$, battery $i$ 's energy value, which means the unit energy value for the BESS, is calculated by dividing the price of the BESS by the total life cycle. It must be set lower than the fuel cost of the diesel generator, to prevent using the diesel generator to increase the SOC.

\subsection{Constraints}

The constraints can be classified into diesel generator-related, BESS-related, and the microgrid overall-related.

When the diesel generator is off $\left(u_{i, t}=0\right)$, the output must be 0 , and when it is on $\left(u_{i, y}=1\right)$, the output must be smaller than the maximum output $g_{i, b}^{M}$.

$$
0 \leq g_{i, t, b} \leq g_{i, b}^{M} \cdot u_{i, t}
$$

Since $s_{i, t}$ and $d_{i, t}$, represent whether the diesel generator is running or stopped, they are closely related to $u_{i, t}$, representing whether the diesel generator is on or off. The relation between these variables should be also represented in a constraint format. The diesel generator's on/off status changes only when it starts to run or stop, using the former status of diesel generator at $t-1$.

$$
u_{i, t}-u_{i, t-1}=s_{i, t}-d_{i, t}
$$

Once the diesel generator starts to run, it has a minimum duration period to stop generating power. To represent such a characteristic, the diesel generator's minimum run time constraint is added as Equation (8). When diesel generator $i$ starts to run $\left(s_{i, t}=1\right)$, it should not stop $\left(d_{i, t}=1\right)$ before it has been running for the minimum run time $\left(M U T_{i}\right)$.

$$
s_{i, t}+d_{i, t}+d_{i, t+1}+\cdots+d_{i, t+M U T_{i}-1} \leq 1
$$

On the other hand, once the diesel generator has stopped generating power, it could run only after a certain period of rest, represented as minimum stop time $\left(M D T_{i}\right)$ in Equation (9).

$$
d_{i, t}+s_{i, t}+s_{i, t+1}+\cdots+s_{i, t+M D T_{i}-1} \leq 1
$$

The status of the diesel generator is represented as $d_{i, t}$, and $s_{i, t}$. Each becomes ' 1 ', when the diesel generator has stopped and is running, respectively.

Must-run or unavailability state is represented as below, by using state variables.

$$
\begin{gathered}
u_{i, t}=0: \text { unavailability } \\
u_{i, t}=1: \text { must }- \text { run }
\end{gathered}
$$


Meanwhile, the pure reserve power $\left(r_{i, t}\right)$, produced by the diesel generator $i$, at time $t$, is only available when it is running. And the amount of reserve power supply from the diesel generator is closely related to the output of the diesel generator. The respective characteristic is represented as below.

$$
\begin{aligned}
& 0 \leq r_{i, t} \leq \mathrm{RC}_{i, t} \cdot u_{i, t} \\
& g_{i, t}+r_{i, t} \leq g_{i}^{M} \cdot u_{i, t} \\
& g_{i, t}-r_{i, t} \geq g_{i}^{m} \cdot u_{i, t}
\end{aligned}
$$

The diesel generator output's increase and decrease ratio is represented as below.

$$
\begin{aligned}
& g_{i, t}-g_{i, t-1} \leq 60 \cdot \mathrm{RUR}_{i} \\
& g_{i, t-1}-g_{i, t} \leq 60 \cdot \mathrm{RDR}_{i}
\end{aligned}
$$

BESS can both charge and discharge energy, so binary variables are necessary to represent whether it is charging or discharging $\left(x_{i, t}, y_{i, t}\right)$, and also the output power $\left(g_{i, t}^{x}, g_{i, t}^{y}\right)$ must be in a constraint format as well. When the BESS is charging, it is handled as a load from the aspect of the power system. Therefore, it must be represented as a negative generation, and the output of the BESS should be shown as below.

$$
\begin{gathered}
g_{i, t}=g_{i, t}^{x}-g_{i, t}^{y} \\
0 \leq g_{i, t}^{x} \leq g_{i}^{X} \cdot x_{i, t} \\
0 \leq g_{i, t}^{y} \leq g_{i}^{Y} \cdot y_{i, t}
\end{gathered}
$$

A binary variable representing whether it is charging or discharging is needed as below.

$$
x_{i, t}+y_{i, t} \leq 1
$$

The energy capacity of BESS $i$, must be in the range of minimum capacity and maximum capacity, which is normally $0(\%)$ and $100(\%)$.

$$
\operatorname{enrg}_{i}^{m} \leq \operatorname{enrg}_{i, t} \leq \operatorname{enrg}_{i}^{M}
$$

In addition, the BESS's initial energy state and final energy demand can be represented as below.

$$
\begin{aligned}
& \operatorname{enrg}_{i, 0}=\operatorname{enrg}_{i}^{0} \\
& \operatorname{enrg}_{i, T}=\operatorname{enrg}_{i}^{T}
\end{aligned}
$$

A constraint format representing the BESS's output-reserve power relation is shown in the following equations.

$$
\begin{aligned}
& 0 \leq r_{i, t} \leq R C_{i, t} \\
& g_{i, t}^{x}+r_{i, t} \leq g_{i}^{X} \\
& g_{i, t}^{y}+r_{i, t} \leq g_{i}^{Y}
\end{aligned}
$$

The power balance qualification must be equal to the summary of total generation and total load $\left(L_{t}\right)$. In order to satisfy the power balance, using the minimum production of the diesel generator and BESS, the remaining demand $\left(D_{t}\right)$ must be applied, excluding the power generation from the wind turbine and photovoltaic device.

$$
\sum_{i} g_{i, t}=D_{T}
$$


A constraint handling the reserve power can be represented by assuming that a certain amount of reserve power must be procured for every time period.

$$
\sum_{i} r_{i, t} \geq R_{t}
$$

\subsection{Considering the Input/Output-Efficiency of PCS Using Piecewise Linearization Method}

As mentioned before, solving UC using MILP results in obtaining the optimal operation schedule of the controllable devices planted in the microgrid. In detail, the output of the diesel generator and BESS at every time step is calculated. From the BESS, the electric power is delivered or received through the PCS. Delivering electric power to the grid refers to discharging the BESS, and receiving refers to charging the BESS. The PCS decides the amount of discharge and charge power and also converts DC to AC or vise-versa, during such procedure, power loss occurs [25]. The loss is related to the efficiency of the designated input/output power value. When the input/output power is small, the efficiency is low and the portion of power loss is high. However, if the input/output power is large, the efficiency is high and the portion of the power loss is low. Such characteristics should be considered while solving UC for an accurate calculation result, and moreover, precise control of the planted devices in the micro rid.

If the power loss occurrence from the PCS is neglected or the efficiency is assumed to be fixed regardless of the input/output amount, the drawn operation schedule causes differences between the calculated and actual performance of the BESS. In addition, using such an inaccurate schedule for the BESS operation as a reference, it makes no reason for the microgrid operator to solve UC for operation scheduling, since the actual operation performance differs from the scheduled reference. An imprecise device operation schedule may cause imbalance between power demand and supply, which can also result in blackouts in severe conditions. In a stand-alone microgrid, the biggest issue is to reduce the difference of renewable energy output and load forecast data, because this causes the largest errors. Therefore, much of the research on microgrid operation focuses on prediction models for weather forecasts, of which the main goal is to calculate a precise operation schedule. Considering the input/output-efficiency characteristics of the PCS is another aspect to achieve for the same goal of increasing the accuracy of the operation schedule.

In this paper, in order to improve the operation schedule accuracy, an additional constraint formula has been added in the MILP calculation process when solving the UC problem. A piecewise linear method to the actual input/output-efficiency characteristic curve of the PCS has been proposed. It has been assumed that the actual input/output-efficiency curve of the PCS, shown as the blue curve from Figure 1, has been piecewise linearized as the black dotted line on Figure 1. The input (kW), output $(\mathrm{kW})$, and efficiency $(\%)$ for each section is in Table 1. Input $(\mathrm{kW})$ refers to the scheduled injection power heading toward the PCS for both when the BESS is charging or discharging. The amount of the injection power is calculated considering the loss from the PCS. For example, if the BESS is scheduled to discharge $50 \mathrm{~kW}$, the input $(\mathrm{kW})$ must be $55.1 \mathrm{~kW}$, meaning the efficiency is $90.8 \%$ for the desired section, causing $5.1 \mathrm{~kW}$ of loss. 


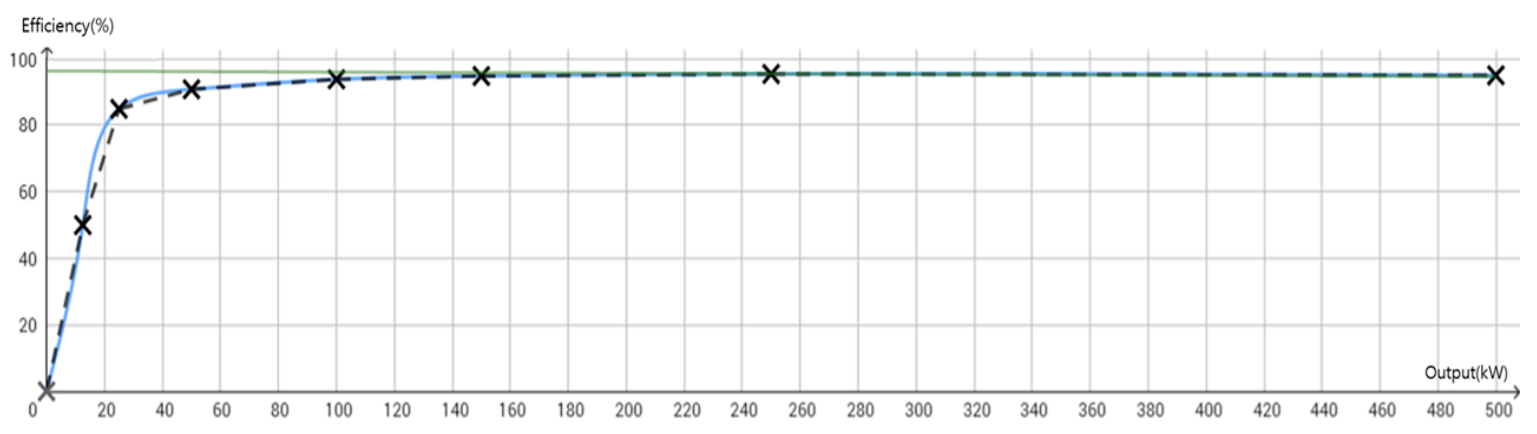

Figure 1. Types of input/output-efficiency characteristic curve of $500 \mathrm{~kW}$ power conditioning system (PCS).

Table 1. Input, output, efficiency for each section of a $500 \mathrm{~kW}$ power conditioning system (PCS).

\begin{tabular}{cccc}
\hline Section & Input (kW) & Output (kW) & Eff (\%) \\
\hline $\mathbf{1}$ & 25.0 & 12.5 & 50 \\
$\mathbf{2}$ & 29.4 & 25 & 84.9 \\
$\mathbf{3}$ & 55.1 & 50 & 90.8 \\
$\mathbf{4}$ & 106.6 & 100 & 93.8 \\
$\mathbf{5}$ & 158.2 & 150 & 94.8 \\
$\mathbf{6}$ & 262.1 & 250 & 95.4 \\
$\mathbf{7}$ & 526.3 & 500 & 95 \\
\hline
\end{tabular}

After applying the piecewise linear method on input/output-efficiency curve of the PCS, an additional formulation is necessary to calculate the input $(\mathrm{kW})$ power of the PCS, considering the related section efficiency, in order to calculate BESS $i^{\prime}$ S SOC $\left(\operatorname{enrg}_{i, t}\right)$. However, the efficiency at a certain section is not equal for when the output $(\mathrm{kW})$ is at minimum or maximum value on the related section discretely, instead it is continual. This continuous relation is represented as the section efficiency slope in Equation (29). The variation of input $(\mathrm{kW})$ and output $(\mathrm{kW})$ is used to define the section efficiency slope. As a result, when solving UC for optimal operation scheduling, the SOC of BESS is calculated using Equation (30), which considers the piecewise linearized input/output-efficiency of the PCS.

$$
\begin{gathered}
\text { slope }_{k}=\frac{\Delta \operatorname{input}(\mathrm{kW})}{\Delta \operatorname{output}(\mathrm{kW})} \\
\text { enrg }_{i, t}=\text { enrg }_{i, t-1}+\left(g_{i, t}^{y}-g_{i, t}^{x}\right) \cdot \text { slope }_{k}
\end{gathered}
$$

\section{Case Study}

This chapter covers the case studies that have been done. Several assumptions are first explained, and the case study results and analysis follow.

\subsection{Assumption}

To verify the proposed stand-alone microgrid UC, a case study was done under an equivalent system environment as Ga-sa Island, South Korea, shown in Figure 2. The load of Ga-sa Island is $83 \sim 107 \mathrm{~kW}$ on average, with a maximum at $167 \mathrm{~kW}$ and minimum at $40 \mathrm{~kW}$. There are three $100 \mathrm{~kW}$ diesel generators, four $100 \mathrm{~kW}$ wind turbines, and a group of photovoltaic devices having total of $320 \mathrm{~kW}$ capacity have been installed. A single $3 \mathrm{MWh}$ Li-ion BESS operating in CVCF mode has been installed, connected by three $500 \mathrm{~kW}$ PCSs, while PCS \#1 is mainly used for normal operation, PCS \#2 and \#3 are used as back-up under emergency situations. For UC calculation, the initial value of BESS SOC is set at $50 \%$, and the value of SOC at the final time period is also set to be $50 \%$ as well. 
In addition, the input/output-efficiency characteristic curve has been piecewise linearized, equal to the black dotted line from Figure 1 and Table 1.

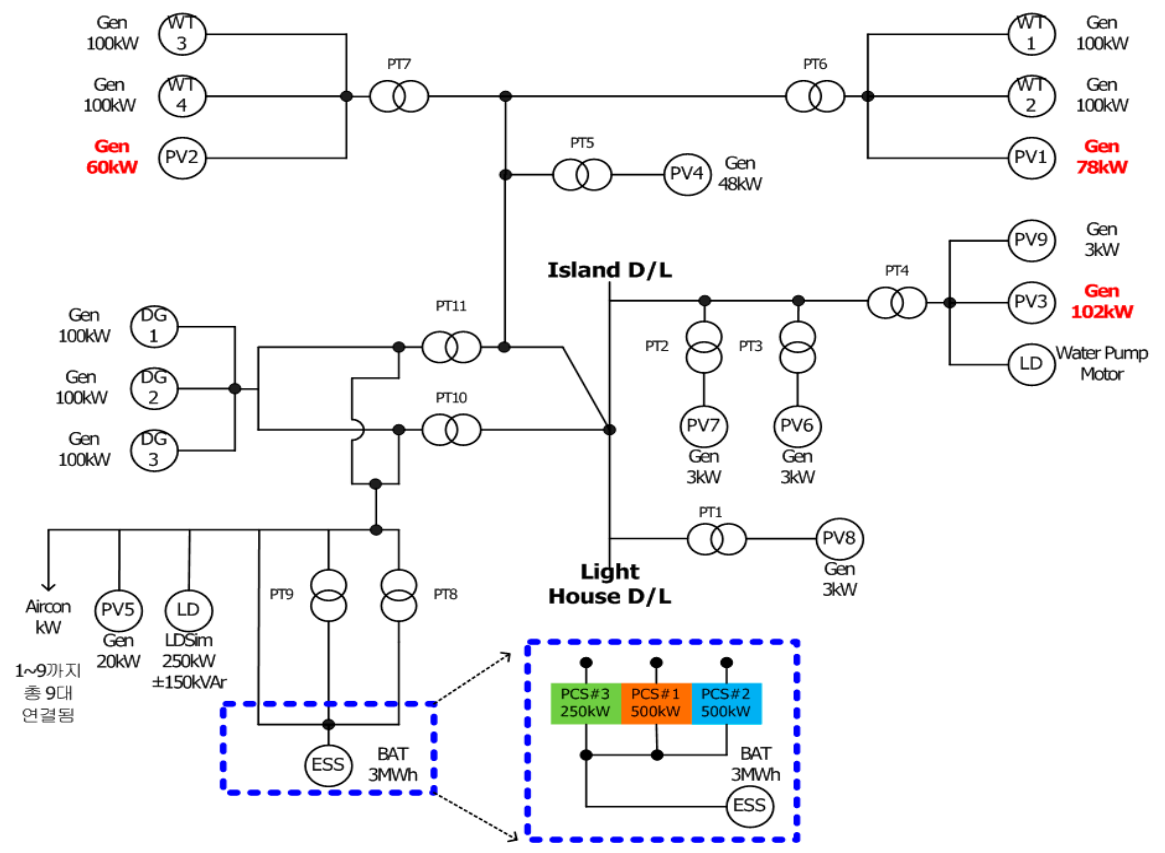

Figure 2. Power System of Ga-sa Island.

The main input data for $\mathrm{UC}$, which is forecast data from the wind turbine and photovoltaic device output and load of Ga-sa Island, is shown in Figure 3. The input forecast data originated from the actual measured data, which was obtained on the 3 April 2015.

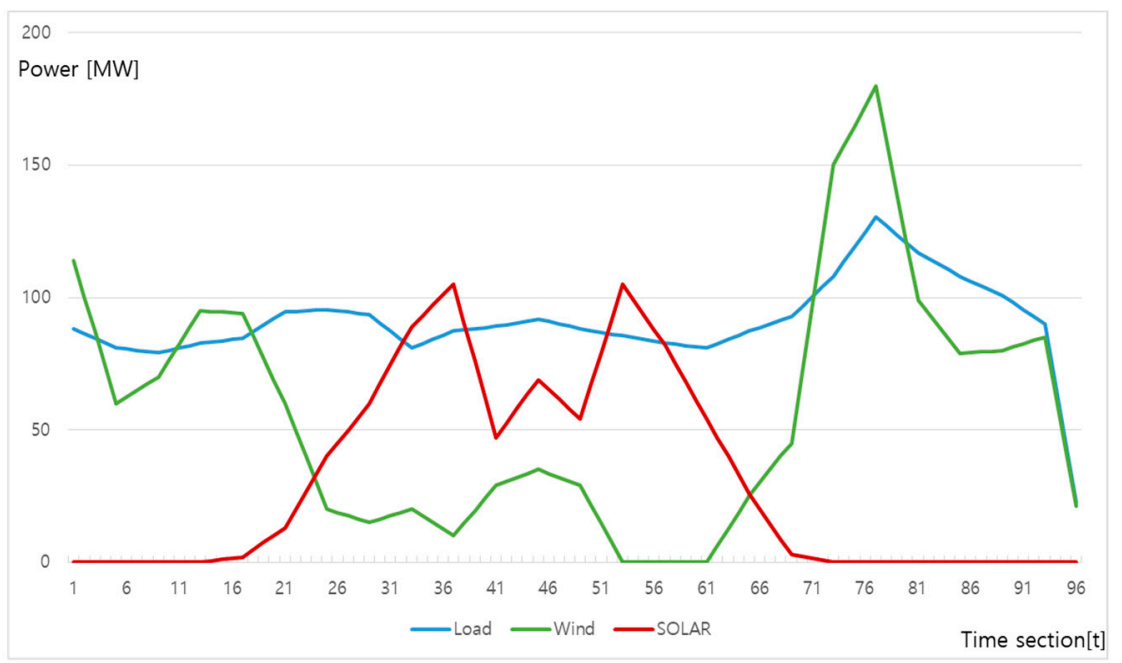

Figure 3. Input forecast data for case study.

To verify the effect of adapting input/output-efficiency of the PCS curve into a linear format by applying a piecewise linear method, a case study was done. Under equal input forecast data and generation device performance, only the efficiency of the PCS is different while solving UC using MILP in the following two cases. CPLEX 2.1 was used as a library function of providing MILP, while the main program was developed using Visual Studio 10. 
Case 1: Assuming fixed PCS efficiency (98\%) regardless of the input/output value (green line from Figure 1).

Case 2: Assuming the input/output-efficiency of the PCS curve to be piecewise linearized (black dotted line from Figure 1).

As a result of calculating UC for both cases, a day-ahead optimal operation schedule for diesel generator output and SOC of the BESS is obtained for a $15 \mathrm{~min}$ time period. Since the input forecast data is equal for both scenarios, the result difference is caused by the input/output-efficiency relation of the PCS. In addition, for each case, the actual power loss from the PCS could be calculated by substituting the scheduled PCS input power for every $15 \mathrm{~min}$ term into the actual input/output-efficiency curve of PCS, the blue line of Figure 1, or by using the following Equation (31). By this method, the power loss could be measured when assuming the microgrid is running on the calculated schedule. Also the amount of power loss from the PCS could be calculated and compared, which enabled the verification of the effect of piecewise linearization to the input/output-efficiency curve of the PCS. Figure 4 shows the analysis process for the case study.

$$
\operatorname{loss}_{p}=\left(g_{i, t}^{x}, g_{i, t}^{y} / e f f_{p}\right) \times 100-g_{i, t}^{x}, g_{i, t}^{y}
$$

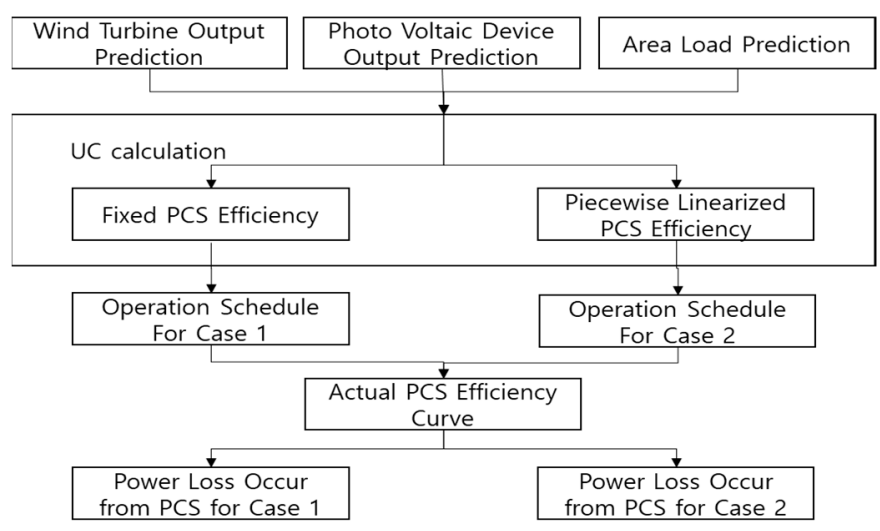

Figure 4. Analysis process for case study.

$\alpha_{i}$, battery $i$ 's energy value is set to be $\$ 3$, after regarding the price of the BESS and its life cycle. Also, the coefficients for the diesel generator, $a_{i}, b_{i}$ and $c_{i}$, are set to be $0.0001,0.2$, and 10, respectively. The fuel cost for the diesel generator is $\$ 1.5$, and the start-up cost is $\$ 4.5$, while the ramp rate for both increase and decrease in generation is set as $7.5 \mathrm{MW} / \mathrm{min}$. $\operatorname{soc}_{i, T}$, the SOC designed to be set at the final schedule time for the BESS, is set to be larger than $50 \% . M U T_{i}$ and $M D T_{i}$, the minimum run time and stop time for the diesel generator, is $0.5 \mathrm{~h}$. The initial energy value of the BESS is assumed to have $50 \%$ of the device's capacity.

\subsection{Case Study Result}

The figures below are the calculation result of the proposed stand-alone microgrid optimal operation schedule by solving UC at the equivalent power system of Ga-sa Island, South Korea.

The red and yellow column from Figures 5 and 6 represent the output from the diesel generator and PCS. When the PCS charges power and stacks SOC for the BESS, it becomes a load for this aspect of the power system. Therefore, the negative expressions in Figures 5 and 6 indicate the charging power from the PCS. The blue line is the SOC. The initial value of SOC is set to be $50 \%$, and the final SOC value is set to be $50 \%$ as well. 


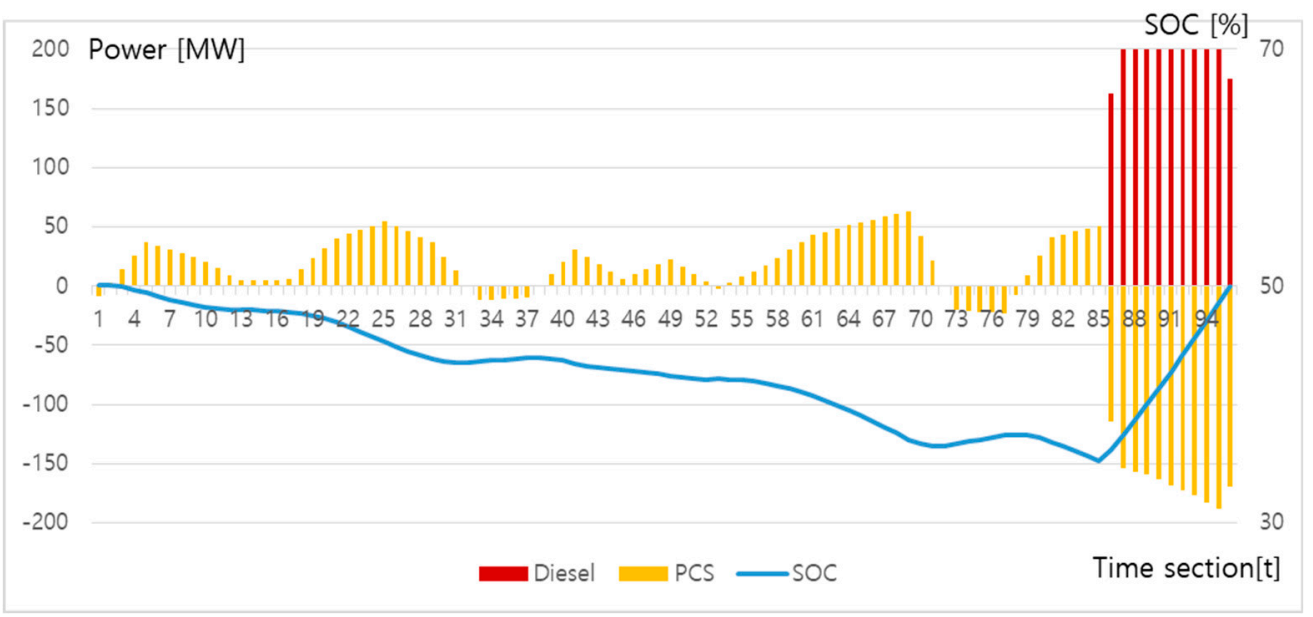

Figure 5. Simulation result from Case 1.

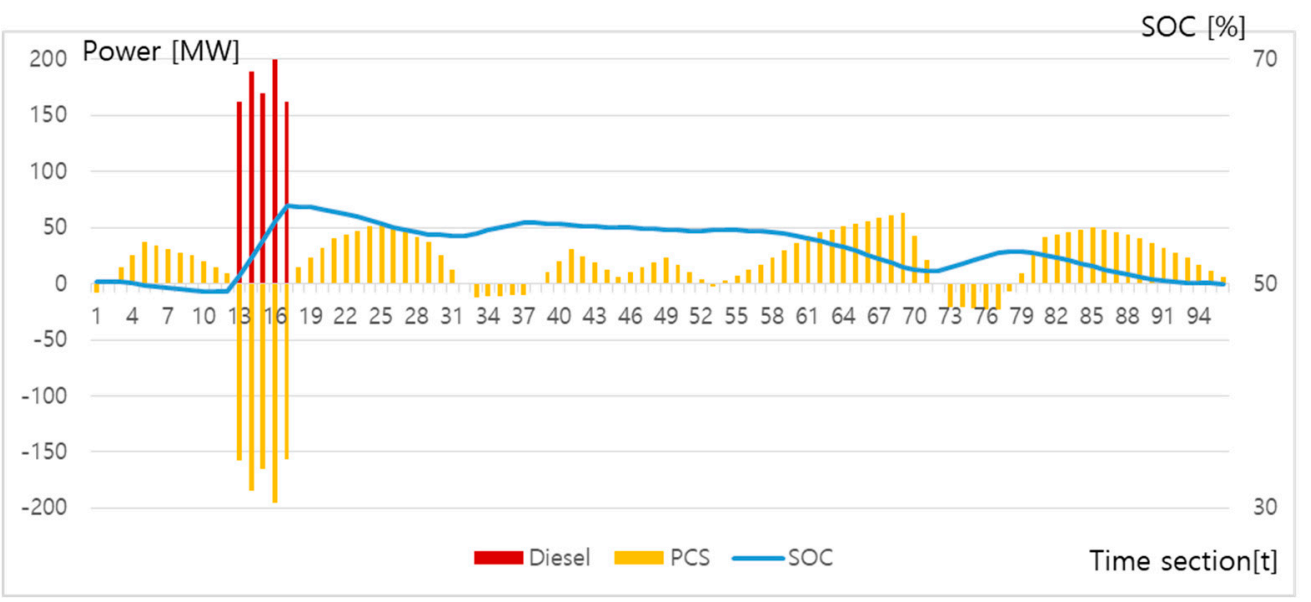

Figure 6. Simulation result from Case 2.

Table 2 is the analysis of case study results. Total generation from diesel and total output from PCS is the summation of each device's calculated result of an optimal operation schedule for 96 time periods. Total loss from PCS is the traced value of loss by substituting each of the calculated optimal operation schedules for the 96 time periods into the actual PCS input/output-efficiency curve.

Table 2. Case Study Analysis.

\begin{tabular}{lccc}
\hline & $\begin{array}{c}\text { Total Generation } \\
\text { from Diesel }[\mathbf{k W h}]\end{array}$ & $\begin{array}{c}\text { Total Output } \\
\text { from PCS [kWh] }\end{array}$ & $\begin{array}{c}\text { Total Loss } \\
\text { from PCS [kWh] }\end{array}$ \\
\hline Case 1 & 2137.4 & -39.8 & 1000.42 \\
Case 2 & 884.1 & 1213.7 & 878.06 \\
\hline
\end{tabular}

The result of UC calculation for Case 1 shows that the diesel generator must produce $2137.4 \mathrm{kWh}$ of electric power in order to satisfy the objective function regarding the constraints. For Case 2, the diesel generator must produce $884.1 \mathrm{kWh}$, which is $1253.3 \mathrm{kWh}$ less than Case 1's result. For the output of the PCS, $-39.8 \mathrm{kWh}$ has been supplied to the microgrid, or $39.8 \mathrm{kWh}$ has been absorbed from the microgrid in Case 1, and for Case 2, $1213.7 \mathrm{kWh}$ has been supplied to the microgrid. The loss incurred from the PCS in Case 1 is $1000.42 \mathrm{kWh}$, and $878.06 \mathrm{kWh}$ for Case 2, which is $122.6 \mathrm{kWh}$ less than Case 1. As expected, one could see that, by calculating the optimal operation schedule while assuming the PCS input/output-efficiency to be fixed, this results in having more loss from the PCS than when the microgrid actually operate as the calculated UC schedule. 
If the actual curve of PCS input/output-efficiency is piecewise linearized as in Case 2, the loss from the PCS could be closer to actual performance. Also, the predicted value of the diesel generator will be calculated more precisely, while maintaining the demand-supply balance for the microgrid. In practice, by comparing the results of Case 1 and Case 2, the diesel generator's generation is reduced by $1253.3 \mathrm{kWh}$, when the piecewise linearized PCS input/output-efficiency is considered, while calculating UC.

\section{Conclusions}

The main goal of this paper is to increase the results of calculated day-ahead schedules for the implanted devices in a stand-alone microgrid. If a CVCF mode BESS is implemented into a stand-alone microgrid, an energy management system is necessary in order to operate the microgrid under stable and economical conditions. In this paper, a UC was proposed for calculating the implemented diesel generator and BESS's optimal operation schedule, based on the forecast data of renewable source generation output and load. To calculate the operation schedule, the summation of generator's start-up cost and fuel cost must be minimized, and many constraints considering the diesel generator and BESS's characteristics should be considered. Also, the calculation result proved to be closer to the actual performance when the input/output-efficiency of the PCS is piecewise linearized and implemented in the UC constraint. This allows one to predict not only the loss from the PCS, but also allows the diesel generator output to be more accurate, compared to when the PCS efficiency is assumed to be fixed. This leads to an improvement in the efficiency and stability of the microgrid's operation. The proposed stand-alone microgrid UC has been simulated and verified under an equivalent power system on Ga-sa Island, South Korea. Any other microgrid in a similar environment could adapt the main idea of this paper.

The frequency of status alteration, such as changing from charging to discharging or vice versa, at the BESS, is a main concern that limits the lifespan of the device. In future research, a formulation to minimize the status alteration of the BESS will be considered and applied to the proposed idea of this paper. With this, it is expected that the lifespan of one of the most expensive electric device used to construct a microgrid can be extended.

Author Contributions: Software, H.-L.L.; Supervision, Y.-H.C.; Writing—original draft, H.-L.L.

Funding: This work was supported by the Energy \& Resource Recycling of the Korea Institute of Energy Technology Evaluation and Planning(KETEP) grant funded by the Korea government Ministry of Knowledge Economy (No. 20171210200830).

Conflicts of Interest: The authors declare no conflict of interest.

\section{Nomenclature}

$\begin{array}{ll}i & \text { subscript representing generator/battery } \in\{1,2, \cdots, \mathrm{I}\} \\ t & \text { subscript representing time } \in\{1,2, \cdots, \mathrm{T}\} \\ b & \text { subscript representing generator's output sec tion } \in\{1,2, \cdots, \mathrm{B}\} \\ a_{i}, b_{i}, c_{i} & \text { coefficient for the diesel generator } i^{\prime} s \text { fuel cost function } \\ u_{i, t} & \text { diesel generator } i^{\prime} s \text { on/off status at time } t \in\{0,1\} \\ s_{i, t} & \text { diesel generator } i^{\prime} s \text { run status at time } t \in\{0,1\} \\ d_{i, t} & \text { diesel generator } i^{\prime} \text { s stop status at time } t \in\{0,1\} \\ x_{i, t} & \text { battery } i^{\prime} s \text { discharge status at time } t \in\{0,1\} \\ y_{i, t} & \text { battery } i^{\prime} s \text { charge status at time } t \in\{0,1\} \\ g_{i, t, b} & \text { diesel generator } i^{\prime} s \text { output at output sec tion b at time } t[\mathrm{MW}] \\ g_{i, t}^{x}, g_{i, t}^{y} & \text { battery } i^{\prime} s \text { discharge/charge output at time } t[\mathrm{MW}] \\ r_{i, t} & \text { diesel generator / battery } i^{\prime} s \text { reserve energy at time } t[\mathrm{MW}] \\ e n r g_{i, t} & \text { battery } i^{\prime} s \text { charging energy at time } t[\mathrm{MWh}] \\ g_{i, t} & \text { diesel generator / battery } i^{\prime} s \text { overall output at time } t[\mathrm{MW}] \\ F L C_{i, t} & \text { diesel generator } i^{\prime} s \text { fuel cos t function }[\$ / \mathrm{h}]\end{array}$




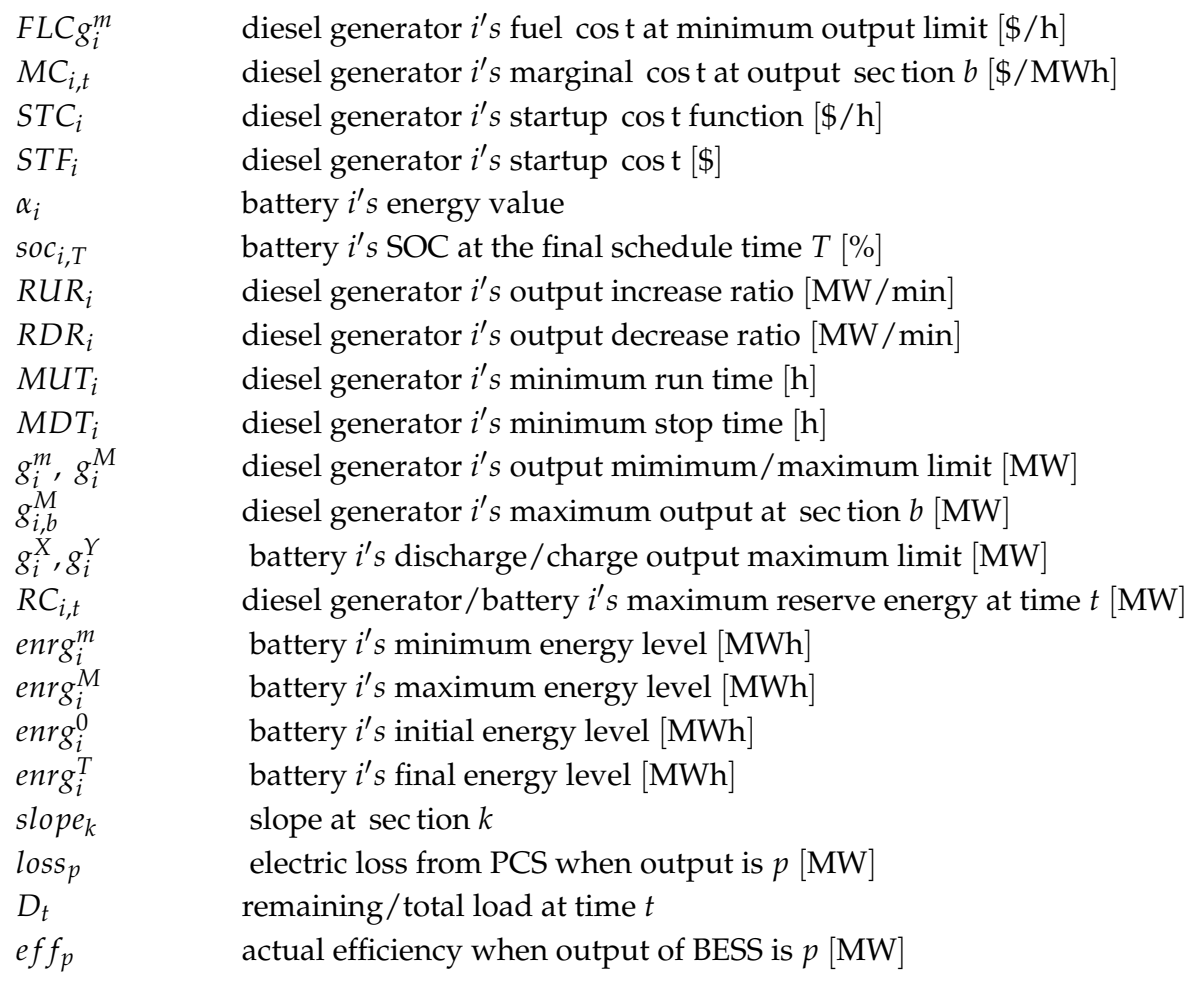

\section{References}

1. Li, X.; Hui, D.; Wu, L.; Lai, X. Control strategy of battery state of charge for wind/battery hybrid power system. In Proceedings of the IEEE International Symposium on Industrial Electronics, Bari, Italy, 4-7 July 2010.

2. Le Dinh, K.; Hayashi, Y. Experiment with an OPF controller based on HPSO-TVAC for a PV-supplied microgrid with BESS. In Proceedings of the IEEE PES General Meeting I Conference \& Exposition, National Harbor, MD, USA, 27-31 July 2014.

3. Katiraei, F.; Iravani, M.R.; Lehn, P. Microgrid autonomous operation during and subsequent to islanding process. In Proceedings of the IEEE Power Engineering Society General Meeting, Denver, CO, USA, 6-10 June 2004.

4. Vandoorn, T.L.; De Kooning, J.D.M.; Meersman, B.; Guerrero, J.M.; Vandevelde, L. Voltage-Based Control of a Smart Transformer in a Microgrid. IEEE Trans. Ind. Electron. 2013, 60, 1291-1305. [CrossRef]

5. Schauder, C.; Mehta, H. Vector analysis and control of advanced static VAr compensators. IEE Proc. C Gener. Transm. Distrib. 1993, 140, 299-306. [CrossRef]

6. Philip, J.; Singh, B.; Mishra, S. Performance evaluation of an isolated system using PMSG based DG set, SPV array and BESS. In Proceedings of the IEEE International Conference on Power Electronics, Drives and Energy Systems (PEDES), Mumbai, India, 16-19 December 2014.

7. Wang, Y.; Tan, K.T.; So, P.L. Coordinated control of battery energy storage system in a microgrid. In Proceedings of the IEEE PES Asia-Pacific Power and Energy Engineering Conference (APPEEC), Kowloon, China, 8-11 December 2013.

8. Piphitpattanaprapt, N.; Bangerdpongchai, D. Optimal dispatch strategy of hybrid power generation with battery energy storage system in islanding mode. In Proceedings of the IEEE Innovative Smart Grid Technologies-Asia (ISGT ASIA), Bangkok, Thailand, 3-6 November 2015.

9. Wu, X.; Wang, X.; Bie, Z. Optimal generation scheduling of a microgrid. In Proceedings of the 3rd IEEE PES Innovative Smart Grid Technologies Europe (ISGT Europe), Berlin, Germany, 14-17 October 2012.

10. Moradi, H.; Esfahanian, M.; Abtahi, A.; Zilouchian, A. Modeling a Hybrid Microgrid Using Probabilistic Reconfiguration under System Uncertainties. Energies 2017, 10, 1430. [CrossRef]

11. Malysz, P.; Sirouspour, S.; Emadi, A. MILP-based rolling horizon control for microgrids with battery storage. In Proceedings of the IECON 2013-39th Annual Conference of the IEEE Industrial Electronics Society, Vienna, Austria, 10-13 November 2013. 
12. Parisio, A.; Rikos, E.; Glielmo, L. A model predictive control approach to microgrid operation optimization. IEEE Trans. Control Syst. Technol. 2014, 22, 1813-1827. [CrossRef]

13. Moshi, G.G.; Bovo, C.; Berizzi, A. Optimal operational planning for pv-wind-diesel-battery microgrid. In Proceedings of the IEEE Eindhoven PowerTech, Eindhoven, The Netherlands, 29 June-2 July 2015.

14. Hu, C.; Luo, S.; Li, Z.; Wang, X.; Sun, L. Energy coordinative optimization of wind-storage-load microgrids based on short-term prediction. Energies 2015, 8, 1505-1528. [CrossRef]

15. Sandhya Rani, P.; Sumanth, K. A Model Predictive Control Approach for Efficient Optimization of Microgrid Operations using Mixed Integer Linear Programming. Int. J. Eng. Res. 2016, 5, 510-524. [CrossRef]

16. Wouters, C.; Fraga, E.S.; James, A.M. An energy integrated, multi-microgrid, milp (mixed-integer linear programming) approach for residential distributed energy system planning-a south Australian case-study. Energy 2015, 85, 30-44. [CrossRef]

17. Zaree, N.; Vahidinasab, V. An MILP formulation for centralized energy management strategy of microgrids. In Proceedings of the Smart Grids Conference (SGC), Kerman, Iran, 20-21 December 2016.

18. Parisio, A.; Glielmo, L. A mixed integer linear formulation for microgrid economic scheduling. In Proceedings of the IEEE International Conference on Smart Grid Communications (SmartGridComm), Brussels, Belgium, 17-20 October 2011; pp. 505-510. [CrossRef]

19. Hawkes, A.D.; Leach, M.A. Modelling high level system design and unit commitment for a microgrid. Appl. Energy 2009, 86, 1253-1265. [CrossRef]

20. Liang, H.Z.; Gooi, H.B. Unit commitment in microgrids by improved genetic algorithm. In Proceedings of the IPEC, Singapore, 27-29 October 2010.

21. Farrokhabadi, M.; Cañizares, C.A.; Bhattacharya, K. Unit Commitment for Isolated Microgrids Considering Frequency Control. IEEE Trans. Smart Grid 2018, 9, 3270-3280. [CrossRef]

22. Deckmyn, C.; Van de Vyver, J.; Vandoorn, T.L; Meersman, B.; Desmet, J.; Vandevelde, L. Day-ahead unit commitment model for microgrids. IET Gener. Transm. Distrib. 2017, 11, 1-9. [CrossRef]

23. Nemati, M.; Braun, M.; Tenbohlen, S. Optimization of unit commitment and economic dispatch in microgrids based on genetic algorithm and mixed integer linear programming. Appl. Energy 2018, 210, 944-963. [CrossRef]

24. Jabbari-Sabet, R.; Moghaddas-Tafreshi, S.M.; Mirhoseini, S.S. Microgrid operation and management using probabilistic reconfiguration and unit commitment. Int. J. Electr. Power Energy Syst. 2016, 75, 328-336. [CrossRef]

25. Kyle, C. Power Conversion System Architectures for Grid Tied Energy Storage; National Institude of Standrads and Technology; Technology Administration, U.S. Department of Commerce: Washington, DC, USA, 2012. 